\title{
Dissolution improvement of solid self-emulsifying drug delivery systems of fenofibrate using an inorganic high surface adsorption material
}

\author{
GAMAL SHAZLY1,2 \\ KAZI MOHSIN $^{+*}$ \\ ${ }^{1}$ Department of Pharmaceutics \\ College of Pharmacy \\ King Saud University \\ Riyadh, Saudi Arabia \\ ${ }^{2}$ Department of Industrial Pharmacy \\ Faculty of Pharmacy \\ Assiut University \\ Assuit, Egypt
}

\begin{abstract}
Solidification of lipid formulations using adsorbents is a recent technique attracting great interest due to its favourable properties including flexibility in dose division, reduction of intra-subject and inter-subject variability, improvement in efficacy/safety profile and enhancement of physical/ chemical stability. The current study aims to convert liquid self-emulsifying/nanoemulsifying drug delivery systems (SEDDS/SNEDDS) into solid SEDDS/SNEDDS and to assess how adsorption of the drug onto an inorganic high surface area material, Neusilin ${ }^{\circledR}$ grade US2 (NUS2), affects its in vitro dissolution performance. Lipid formulation classification systems (LFCS) Type III formulations were designed for the model anti-cholesterol drug fenofibrate. NUS2 was used to solidify the SEDDS/SNEDDS. Particle size and SEM analyses of solid SEDDS/SNEDDS powder were carried out to investigate the adsorption efficiency. In vitro dissolution studies were conducted to compare the developed formulations with the marketed product. The results of characterization studies showed that the use of $50 \%(\mathrm{~m} / \mathrm{m})$ adsorbent resulted in superior flowability and kept the drug stable is amorphous state. Dissolution studies allow the conclusion that the formulation containing a surfactant of higher water solubility (particularly, Type IIIB SNEDDS) has comparably faster and higher release profiles than Type IIIA (SEDDS) and marketed product.
\end{abstract}

Keywords: self-emulsifying/nanoemulsifying drug delivery systems, fenofibrate, in vitro dissolution test, solidification technique, adsorption

Lipid-based dosage forms have gained high priority and become more prominent in the pharmaceutical industry in recent years (1-4). Lipids have been used as carriers in various delivery systems for drug administration, including solutions, suspensions, emulsions, self-emulsifying systems, microemulsions and, recently, solid dosage forms (via

\footnotetext{
*Correspondence; e-mail: mkazi@ksu.edu.sa
} 
technology conversion) such as solid self-emulsifying systems. They are designed to increase the solubility and bioavailability of drugs belonging to the BCS classes II and IV, in which the dissolution rate is the limiting factor for absorption (5-8). Lipid-based carriers are usually liquid at room temperature and are generally encapsulated into soft/hard gelatin capsules for final oral delivery. This could be a great challenge since the interaction between the liquid formulation and capsule shell may result in either brittleness or softness of the shell (9). In addition, the stability of liquid formulations could be another major issue (e.g. leaching and rancidity), since some drugs might suffer significant chemical instability in both aqueous and oily formulations.

To address this limitation, incorporation of liquid lipid formulations into a solid dosage form is therefore convincing and desirable. Liquid lipid formulations could be transformed into acceptable free flowing powder by loading the formulation onto a suitable solid carrier (10, 11). Solid dispersions and in particular self-emulsifying drug delivery systems (SEDDS) have proved to be the most practical route to commercialization. Success

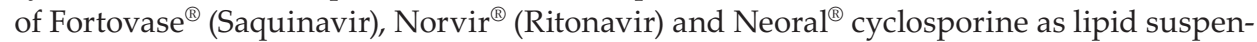
sions have prompted formulators to develop more stable forms by converting these suspensions into powder (12). Neusilin ${ }^{\circledR \prime}$ 's high specific area, increased surface adsorption, porosity, anticaking, flow enhancing properties and its ability to keep the drug stable under amorphous state make it one of the best choices among the adsorbents available today (13). Good understanding of the characteristics of lipid excipients and their performance in formulations is desirable to rank and select suitable excipients in formulation development.

Fenofibrate is a lipid-regulating agent that has chemical, pharmacological, and clinical similarities to other fibrate drugs such as clofibrate and gemfibrozil (14). Fenofibrate is a BCS class II drug with a high dose number (15). It can be assumed that the low oral bioavailability of fenofibrate is due to its solubility and dissolution limitations. It is a nonelectrolyte with low aqueous solubility $\left(<3 \mu \mathrm{g} \mathrm{mL} \mathrm{m}^{-1}\right)$ and fairly high octanol/water partition coefficient $(\log P$ 4.6).

Efforts to develop tablet formulations containing sufficient quantities of lipid and surfactant excipients to solubilize poorly soluble drugs have met with limited success due to the tendency of these excipients to compromise the physical integrity and mechanical strength of conventional compressed tablets. To achieve extensive absorption of a poorly water soluble compound from the GI tract requires an innovative formulation approach. Encapsulation of formulations such as liquids or semi-solids provides opportunities for delivering poorly water-soluble compounds with reproducible absorption and acceptable bioavailability.

While an enormous amount of industrial interest exists in transforming such liquid lipid formulations into free-flowing powders that are compatible with direct tablet pressing, only a limited number of studies have been conducted to investigate the solidification of lipid formulations; they have been focused on their adsorption onto inorganic high surface area materials. The goal of the current research is to assess how the adsorption of drug loaded SEDDS/SNEDDS onto an inorganic high surface area material (Neusilin ${ }^{\circledR}$ grade US2) affects its in vitro (dissolution) performance. 


\section{EXPERIMENTAL}

\section{Materials}

All chemicals used in the studies were obtained from commercial suppliers. Fenofibrate (2-[4-(4-chlorobezoyl) phenoxy]-2-methylpropionic acid 1-methylethyl ester) was supplied by Sigma-Aldrich Co, USA. Miglyol 812 (M812), Imwitor 988 (I988), Imwitor 308 (I308) and propylene glycol (PG, 98 \% pure) were supplied by Sasol Germany GmbH, Germany. The non-ionic surfactants used were TO-106V (PEG 6 sorbitan monooleate, HLB-10) and HCO-30 (PEG 30 hydrogenated castor oil, HLB-11), supplied by Nikko Chemicals Co., Japan. All excipients were used without further purification. Cremophor EL (CrEL, polyoxyethylene 35 castor oil), and Cremophor RH40 (CrRH40, polyoxyethylene 40 castor oil) were from Sigma Chemical Co. (USA). Neusilin ${ }^{\circledR}$ grade US2 (NUS2, synthetic magnesium aluminometasilicate, Fuji Chemical Co., Japan) was used as the microporous adsorbent to load SEDDS/SNEDDS. Lipanthyl ${ }^{\circledR}$ (marketed product of fenofibrate, $200 \mathrm{mg}$ micronized capsule) manufactured by Abbott (USA) was used in the study. Water used in this study was obtained from a Milli-Q water purification system (Sartorius, Germany). All other chemicals and solvents were of analytical purity.

\section{Methods}

Design of liquid SEDDS/SNEDDS. - Within the current scope of the study, self-emulsifying lipid formulations, particularly SEDDS/SNEDDS, have been developed using lipid formulation classification systems (LFCS). LFCS was proposed by Pouton in 2000 in four categories (16) to gain knowledge of excipient selection and compare the performance of various lipid-based drug delivery systems. Hence, LFCS type IIIA and IIIB lipid-based formulations (SEDDS/SNEDDS) have been prepared in two component systems with varying oil and surfactant concentrations. For systems which involved a blend of two oils with a surfactant, the strategy was to weigh and combine a primary mixture of the two oil components. For example, a mixture of M812 and I988 (7:3, by mass) was prepared and then blended with $50 \%$ surfactant. The mixture was thoroughly blended with a Vm1 vortex (Ratek Instruments Pty. Ltd., Victoria, Australia), with heating if necessary, to ensure uniformity. The formulations were prepared using 5-g capacity vials and were tightly sealed until further use.

Droplet size distribution of liquid SEDDS/SNEDDS. - The droplet size distribution of diluted SEDDS/SNEDDS was measured by laser diffraction analysis using Zetasizer (Nano ZS, Germany) particle sizing systems (17). The formulations were diluted at a ratio of 1:1000 V/V (SEDDS/SNEDDS : distilled water) and mixed for $1 \mathrm{~min}$ before analysis. Samples were placed directly into the module and measured 10 times. Particle size was calculated from the volume size distribution. All experiments were performed in triplicate and good agreement was found between measurements. As reported earlier, the self-emulsifying efficiency is strongly associated with the mean droplet size of the produced emulsion (18, 19).

Preparation of solid SEDDS/SNEDDS. - The liquid SEDDS/SNEDDS was added gradually and blended with the adsorbent NUS2 at the following fixed final SEDDS/SNEDDS to adsorbent appropriate mass ratio of 50/50, 60/40 and 70/30 $(\%, \mathrm{~m} / \mathrm{m})$. Briefly, a fixed amount 
of liquid SEDDS/SNEDDS was initially added to and mixed with NUS2 (20). The batch size of each blend was varied according to the quantity of the adsorbent and was categorized based on the flowability of the powder. The flow behaviour of the adsorbed blend was assessed visually only to measure if a non-flowing cohesive mass was formed.

Characterization of solid SEDDS/SNEDDS powder. - Particle size, shape and bulk density affect the flowability of powder material. The angle of repose for the solid SEDDS/ SNEDDS was measured to determine the flowability of the powder (21). Briefly, a glass funnel was fitted at a fixed height $(H)$ and its tip was distanced from wax paper placed on a horizontal surface. Solid powder was transferred through the funnel to the surface until the apex of the conical pile reached the tip of the funnel. The test result was calculated as follows:

$$
\text { angle of repose }=\tan \alpha=H / r
$$

where $r$ is the radius of the conical pile and $\alpha$ is the angle of repose.

Particle size determination of solid SEDDS/SNEDDS. - Size distribution of the drug loaded solid SEDDS/SNEDDS powder was investigated using laser light diffraction (Mastersizer Scirocco 2000, Malvern Instruments, UK). For a typical experiment, about $300 \mathrm{mg}$ of the drug loaded solid SEDDS/SNEDDS powder was fed into the sample micro feeder. All samples were analyzed 5 times and average results were taken. The sizes below $10 \%$ $[d(0.1)], 50 \%[d(0.5)]$ and $90 \%[d(0.9)]$ of the pellet were used to characterize the pellets size distribution. The mean diameter was taken as the average of $d(0.1), d(0.5)$, and $d(0.9)$ values.

$X$-ray diffraction (XRD). - Powder crystallinity was assessed by a multipurpose X-ray diffractometer (Ultima IV, Rigaku, Japan). The samples were analyzed using CuK $\alpha$ radiation of wavelength $1.54056 \AA$, generated at $40 \mathrm{kV}$ voltage, $40 \mathrm{~mA}$ current and receiving slit of $0.3 \mathrm{~mm}$. Analyses were preformed over $2 \theta$ range of $3-60^{\circ}$ with an angular increment of $0.5^{\circ} \mathrm{min}$ and scan step time of $1.0 \mathrm{~s}$.

Differential scanning calorimetry (DSC). - DSC analyses of powdered samples were conducted using DSC-60, Shimadzu, Japan. The samples (3-5 mg) were hermetically sealed in aluminum pans and heated at a scanning rate of $10{ }^{\circ} \mathrm{C} \mathrm{min}-1$ under dry nitrogen flow (30 $\mathrm{mL} \mathrm{min}^{-1}$ ) over a temperature range of $0-200{ }^{\circ} \mathrm{C}$. Pure water and indium standard were used to calibrate the DSC temperature and enthalpic scale. Data from the thermal analysis were recorded using the Shimadzu software program in a TA 50I PC system.

Scanning electron microscopy (SEM). - Surface morphology of the raw drug, NUS2 and solid SEDDS/SNEDDS powder was visualized by SEM (Jeol JSM 5400LV SEM, Japan) operated at $15 \mathrm{kV}$. The samples were sputter-coated with a thin gold (SPI sputter coater, PA19380, USA) palladium layer under an argon atmosphere in a high vacuum evaporator and images were then acquired from the system.

In vitro dissolution studies. - The in vitro dissolution studies of the representative solid SEDDS/SNEDDS powder were filled in capsules (size 0) for the experiment. Dissolution of solid SEDDS/SNEDDS, pure fenofibrate powder and the marketed fenofibrate capsule (200 mg Lipanthyl) was monitored using an automated dissolution tester (Logan Instrument Corp, USA) coupled to an automated sample collector (SP-100 peristaltic pump Logan). The standard USP II (apparatus 2) (paddle type, Model UDT-804, Logan) paddle method was used at $50 \mathrm{rpm}$ and $37 \pm 0.5^{\circ} \mathrm{C}$. Dissolution media $(900 \mathrm{~mL})$ of $\mathrm{pH} 1.2$ and $\mathrm{pH} 7.4$ were selected to mimic the physiological $\mathrm{pH}$ of the GIT. Samples were collected periodically after 
$15,30,45,60,90$, and 120 min and replaced by freshly prepared dissolution medium. After filtration through a microfilter $(0.45 \mu \mathrm{m})$, fenofibrate in the withdrawn samples was analyzed at $288 \mathrm{~nm}$ by UHPLC (Dionex, ultimate 3000, Thermo Scientific, USA) using an appropriate solvent. Chromatographic separation was performed on a reversed-phase Acquity ${ }^{\circledR} \mathrm{BEH} \mathrm{C}_{18}$ column $\left(1.7 \mu \mathrm{m}\right.$ particle size, $50 \mathrm{~mm} \times 2.1 \mathrm{~mm}$ ID) at $30{ }^{\circ} \mathrm{C}$ with an isocratic elution profile. The mobile phase consisting of ethanol and water $(70: 30, \%, V / V)$ at a flow rate of $0.3 \mathrm{~mL} \mathrm{~min}^{-1}$ was used. All experiments were carried out in triplicate.

\section{RESULTS AND DISCUSSION}

Composition of lipid-based SEDDS/SNEDDS systems using various concentrations of oil and surfactant are presented in Table I. There are most interesting formulation systems within the lipid formulation classification systems (LFCS), which are likely to produce nanoparticle formulations.

Table I. Oil and surfactant composition of liquid SEDDS/ SNEDDS formulations

\begin{tabular}{|c|c|c|c|c|c|c|c|}
\hline \multirow{2}{*}{$\begin{array}{l}\text { LFCS } \\
\text { type }\end{array}$} & \multicolumn{3}{|c|}{ Oil $(\%, m / m)$} & \multicolumn{4}{|c|}{ Surfactant $(\%, m / m)$} \\
\hline & M812 & I988 & I308 & TO106V & HCO30 & CrEL & CrRH40 \\
\hline IIIA & 35 & 15 & & 50 & & & \\
\hline IIIA & 35 & 15 & & & 50 & & \\
\hline IIIA & 35 & 15 & & & & 50 & \\
\hline IIIA & 35 & 15 & & & & & 50 \\
\hline IIIB & & & 50 & & 50 & & \\
\hline IIIB & & & 50 & & & 50 & \\
\hline IIIB & & & 50 & & & & 50 \\
\hline $\begin{array}{l}\text { Chemical } \\
\text { property }\end{array}$ & $\begin{array}{l}\text { Medium } \\
\text { chain } \\
\text { triglyceride }\end{array}$ & $\begin{array}{l}\text { Medium } \\
\text { chain } \\
\text { mono and } \\
\text { diglyceride }\end{array}$ & $\begin{array}{l}\text { Medium } \\
\text { chain } \\
\text { mono } \\
\text { glyceride }\end{array}$ & $\begin{array}{c}\text { PEG } 6 \\
\text { sorbitan } \\
\text { monooleate }\end{array}$ & $\begin{array}{l}\text { PEG } 30 \\
\text { castor oil }\end{array}$ & $\begin{array}{l}\text { POE } 40 \\
\text { castor oil }\end{array}$ & $\begin{array}{c}\text { POE } 35 \\
\text { castor oil }\end{array}$ \\
\hline
\end{tabular}

a Designed according to lipid formulation classification systems (LFCS).

POE - polyoxyethylene

PEG - polyethyleneglycol

Droplet size analysis of liquid SEDD. - Self-emulsification of mixtures containing medium chain mono/di/tri-glycerides [M812:I988 (7:3)] with water insoluble surfactant (TO$106 \mathrm{~V}$ and/or HCO-30) showed the particle size above $50 \mathrm{~nm}$, and were thus represented as SEDDS (Table II). On the other hand, similar oil compositions [M812:1988 (7:3)] with water soluble surfactant (CrEL and or CrRH40) showed a particle size lower than $50 \mathrm{~nm}$, and were represented as SNEDDS (Table II). The apparent particle size distributions of polar oil I308 with all the representative surfactants in the formulations were found to be lower than $50 \mathrm{~nm}$ (Table II), producing nanodispersions at surfactant concentrations of $50 \%$; the dispersion nature of all the formulations was homogeneous upon aqueous dilution even though SNEDDS3 and SNEDDS4 produced more heterogeneous particles, which was shown by the higher PDI values (see Table II). However, oil and surfactant used in both 
G. Shazly and K. Mohsin: Dissolution improvement of solid self-emulsifying drug delivery systems of fenofibrate using an inorganic high surface adsorption material, Acta Pharm. 65 (2015) 29-42.

Table II. Mean particle size of the drug loaded liquid SEDDS/SNEDDS of different types of formulations

\begin{tabular}{llccc}
\hline System & Formulation composition & $\begin{array}{c}\text { Sol 80 \% } \\
\left(\mathrm{mg} \mathrm{g}^{-1}\right)^{\mathrm{a}}\end{array}$ & $\begin{array}{c}\text { Particle size } \\
(\mathrm{nm})^{\mathrm{b}}\end{array}$ & pdic $^{\mathrm{c}}$ \\
\hline SEDDS1 & M812:I988(7:3)/TO-106V (1/1) & 106.85 & 219.90 & 0.266 \\
SEDDS2 & M812:I988(7:3)/HCO-30 (1/1) & 113.04 & 66.97 & 0.138 \\
SNEDDS1 & M812:I988(7:3)/CrEL (1/1) & 116.80 & 39.90 & 0.076 \\
SNEDDS2 & M812:I988(7:3)/CrRH40 (1/1) & 112.30 & 40.27 & 0.350 \\
SNEDDS3 & I308/HCO-30 (1/1) & 81.90 & 50.29 & 0.594 \\
SNEDDS4 & I308/CrEL (1/1) & 85.59 & 36.03 & 0.702 \\
SNEDDS5 & I308/CrRH40 (1/1) & 80.18 & 49.05 & 0.222 \\
\hline
\end{tabular}

${ }^{c}$ pdi - polydispersity index is a measure of the heterogeneity of sizes of particles in a mixture.

${ }^{\mathrm{b}}$ Mean, $n=3$; RSD $<5 \%$.

a Fenofibrate was loaded in the formulations at $80 \%$ of equilibrium solubility.

SEDDS and SNEDDS produced the finest emulsions but a limited particle size difference between them is not likely to determine the differences between their performances in vivo.

\section{Analyses of solid SEDDS}

Particle size determination of solid SEDDS. - The z-average diameter and volume mean distribution of the solid SEDDS/SNEDDS powder, according to the percentage of adsorbent NUS2 added in the liquid formulations, are presented in Table III. The z-average diameter was measured after the solid powder was dispersed in water and then filtered (using a $0.22-\mu \mathrm{m}$ filter). In most of the formulation systems, the particle size was reduced with the increase in NUS2 content. In addition, it was found by visual determination that the use of $50 \%$ NUS2 showed good flowability of the powders.

Measurement of the angle of repose. - In the current studies, ratios of liquid SEDDS/ SNEDDS to NUS2 of 1:1, 1.5:1, and 2.3:1 produced dry free flowing powders. There were no substantial changes between these ratios due to the high adsorption capacity of the adsorbent (NUS2) and retention of liquid SEDDS/SNEDDS in their intra-particular pores. On visual inspection, all powders were dry and free flowing at this point. However, the liquid SEDDS/SNEDDS-to-NUS2 ratios of 1:1 and 1.5:1 were better than 2.3:1 (Table IV). This suggests that the selection of 1.5:1 ratio would be ideal for good flowability of the solid SEDDS/ SNEDDS powder and high drug loading for fenofibrate.

$X$-ray diffraction analysis. - XRD studies were performed to justify the physical nature of the drug in solid SEDDS/SNEDDS formulations. Fig. 1 depict the XRD spectra of pure fenofibrate, NUS2 and the representative solid SEDDS/SNEDDS formulations. Pure fenofibrate showed sharp peaks in the spectra, which were obtained at 13.79, 15.21, 20.05, 22.58, 31.76 , and $50.12{ }^{\circ} \mathrm{C}(2 \theta)$, due to the native crystalline form of the drug. There were no peaks in NUS2 and representative SEDDS/SNEDDS formulations. Hence, the disappearance of drug peaks in the representative solid SEDDS/SNEDDS formulations established the presence of fenofibrate in amorphous form.

Differential scanning calorimetry (DSC). - The generated DSC thermograms of pure fenofibrate, adsorbent NUS2, and the representative solid SEDDS/SNEDDS formulations are 
G. Shazly and K. Mohsin: Dissolution improvement of solid self-emulsifying drug delivery systems of fenofibrate using an inorganic high surface adsorption material, Acta Pharm. 65 (2015) 29-42.

Table III. Particle size measurement of drug loaded solid SEDDS/SNEDDS powder and dispersant ${ }^{a}$

\begin{tabular}{|c|c|c|c|}
\hline Formulation & $\begin{array}{c}\text { NUS2 } \\
(\%, m / m)\end{array}$ & $\begin{array}{l}\text { Particle size of dispersant } \\
(\mathrm{nm})^{\mathrm{c}}\end{array}$ & $\begin{array}{l}\text { Particle size of powder } \\
(\mathrm{nm})^{\mathrm{c}}\end{array}$ \\
\hline \multirow{3}{*}{$\begin{array}{l}\text { M812:I988(7:3)/TO-106V } \\
\text { (1/1)-SEDDS1 }\end{array}$} & 30 & 227.90 & 561.78 \\
\hline & 40 & 181.70 & 570.72 \\
\hline & 50 & 176.20 & 515.18 \\
\hline \multirow{3}{*}{$\begin{array}{l}\text { M812:1988(7:3)/HCO-30 } \\
\text { (1/1)- SEDDS2 }\end{array}$} & 30 & 129.89 & 835.41 \\
\hline & 40 & 118.75 & 816.54 \\
\hline & 50 & 115.34 & 651.60 \\
\hline \multirow{3}{*}{$\begin{array}{l}\text { M812:I988(7:3)/CrEL } \\
\text { (1/1)- SNEDDS1 }\end{array}$} & 30 & 118.41 & 681.35 \\
\hline & 40 & 98.35 & 769.13 \\
\hline & 50 & 95.31 & 592.87 \\
\hline \multirow{3}{*}{$\begin{array}{l}\text { M812:I988(7:3)/CrRH40 } \\
\text { (1/1)- SNEDDS2 }\end{array}$} & 30 & 168.68 & 751.98 \\
\hline & 40 & 157.13 & 803.34 \\
\hline & 50 & 134.66 & 846.87 \\
\hline \multirow{3}{*}{$\begin{array}{l}\text { I308/HCO-30 } \\
(1 / 1)-\text { SNEDDS3 }\end{array}$} & 30 & 98.80 & 913.21 \\
\hline & 40 & 76.95 & 921.58 \\
\hline & 50 & 75.81 & 739.03 \\
\hline \multirow{3}{*}{$\begin{array}{c}\text { I308/CrEL } \\
(1 / 1)-\text { SNEDDS4 }\end{array}$} & 70 & 77.45 & 857.63 \\
\hline & 60 & 60.37 & 876.78 \\
\hline & 50 & 54.89 & 787.44 \\
\hline \multirow{3}{*}{$\begin{array}{l}\text { I308/CrRH40 } \\
\text { (1/1)- SNEDDS5 }\end{array}$} & 70 & 65.56 & 907.84 \\
\hline & 60 & 59.23 & 767.58 \\
\hline & 50 & 54.35 & 571.54 \\
\hline
\end{tabular}

a Liquid SEDDS/SNEDDS, 1:1000 dilutions in water.

${ }^{\mathrm{b}}$ NUS2 was used at various ratios in the lipid formulation and fenofibrate was loaded at $80 \%$ of equilibrium solubility.

${ }^{\mathrm{c}}$ Mean, $n=3$; RSD $<10 \%$.

Table IV. Flow properties of powders after adsorption of liquid formulations onto Neusilin ${ }^{\circledR}$ US2 at various ratios

\begin{tabular}{cccc}
\hline Liquid formulation $^{\mathrm{a}}$ & Liquid formulation : NUS2 & Angle of repose & Flowability \\
\hline F1 & $1: 1$ & 32.8 & good \\
F2 & $1.5: 1$ & 34.5 & good \\
F3 & $1: 1$ & 33.5 & good \\
F4 & $1.5: 1$ & 38.5 & fair \\
F5 & $1: 1$ & 34.5 & good \\
F6 & $1.5: 1$ & 37.5 & fair \\
F7 & $2.3: 1$ & 42 & pass. \\
F8 & $2.3: 1$ & 42 & pass. \\
F9 & $2.3: 1$ & 42 & pass. \\
\hline
\end{tabular}

${ }^{a}$ F1/F2/F7 - liquid formulation of SEDDS1, F3/F4/F8 - liquid formulation of SEDDS2, F5/F6/F9 - liquid formulation of SNEDDS3 
G. Shazly and K. Mohsin: Dissolution improvement of solid self-emulsifying drug delivery systems of fenofibrate using an inorganic high surface adsorption material, Acta Pharm. 65 (2015) 29-42.

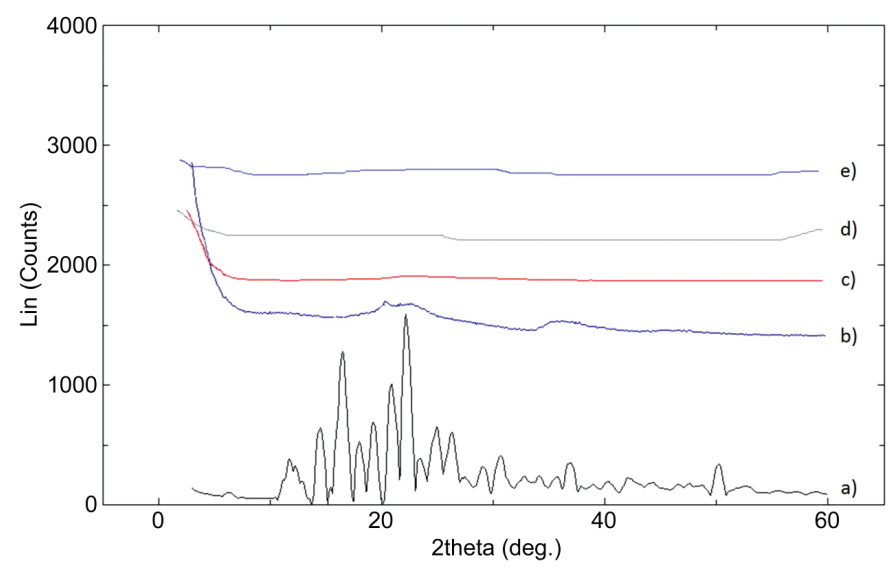

Fig. 1. X-ray powder diffraction spectra of: a) pure fenofibrate, b) Neusilin US2, c) solid SEDDS-1/ NUS2 50:50\% $(\mathrm{m} / \mathrm{m})$, d) solid SEDDS-2/NUS2 50:50 \% $(\mathrm{m} / \mathrm{m})$, e) solid SNEDDS-3/NUS2 50:50 \% $(\mathrm{m} / \mathrm{m})$.

shown in Fig. 2. A sharp endothermic peak of pure fenofibrate was observed at $81.25^{\circ} \mathrm{C}$. However, there were no peaks for NUS2 and solid SEDDS/SNEDDS formulations. This suggests that fenofibrate converted from crystalline to amorphous state during the preparation of solid SEDDS/SNEDDS using NUS2.

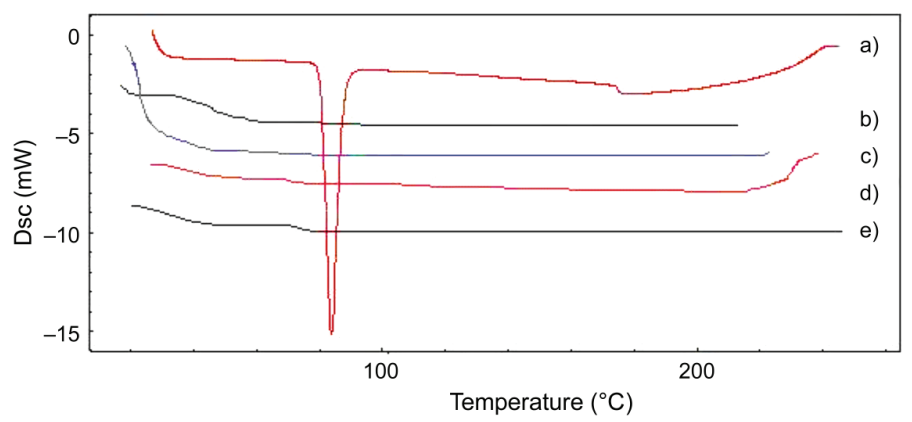

Fig. 2. DSC thermograms of: a) pure fenofibrate, b) pure adsorbent NUS2, c) solid SEDDS-1/NUS2 50:50 \% $(\mathrm{m} / \mathrm{m})$, d) solid SEDDS-2/NUS2 50:50 \% $(\mathrm{m} / \mathrm{m})$, e) solid SNEDDS-3/NUS2 50:50 \% $(\mathrm{m} / \mathrm{m})$.

Scanning electron microscopy. - The scanning electron micrographs in Fig. 3a showed fenofibrate as a crystalline powder with irregularly shaped crystals. Surface morphology of the NUS2 and solid SEDDS/SNEDDS formulations shown in Figs. 3b-e was found homogeneous, uniform and smooth.

In vitro dissolution studies. - In vitro release profiles of fenofibrate from the representative solid SEDDS-1 [M812:I988 (7:3)/TO-106V (1/1)], SEDDS-2 [M812:I988 (7:3)/HCO-30 (1/1)], and SNEDDS3 [I308/HCO30 (1/1)], control (fenofibrate raw powder) and marketed product (Lipanthyl ${ }^{\circledR}$ ) in $\mathrm{HCl}(\mathrm{pH} 1.2)$ at $37^{\circ} \mathrm{C}$ are shown in Fig. 4. It is evident that the percentage 
G. Shazly and K. Mohsin: Dissolution improvement of solid self-emulsifying drug delivery systems of fenofibrate using an inorganic high surface adsorption material, Acta Pharm. 65 (2015) 29-42.
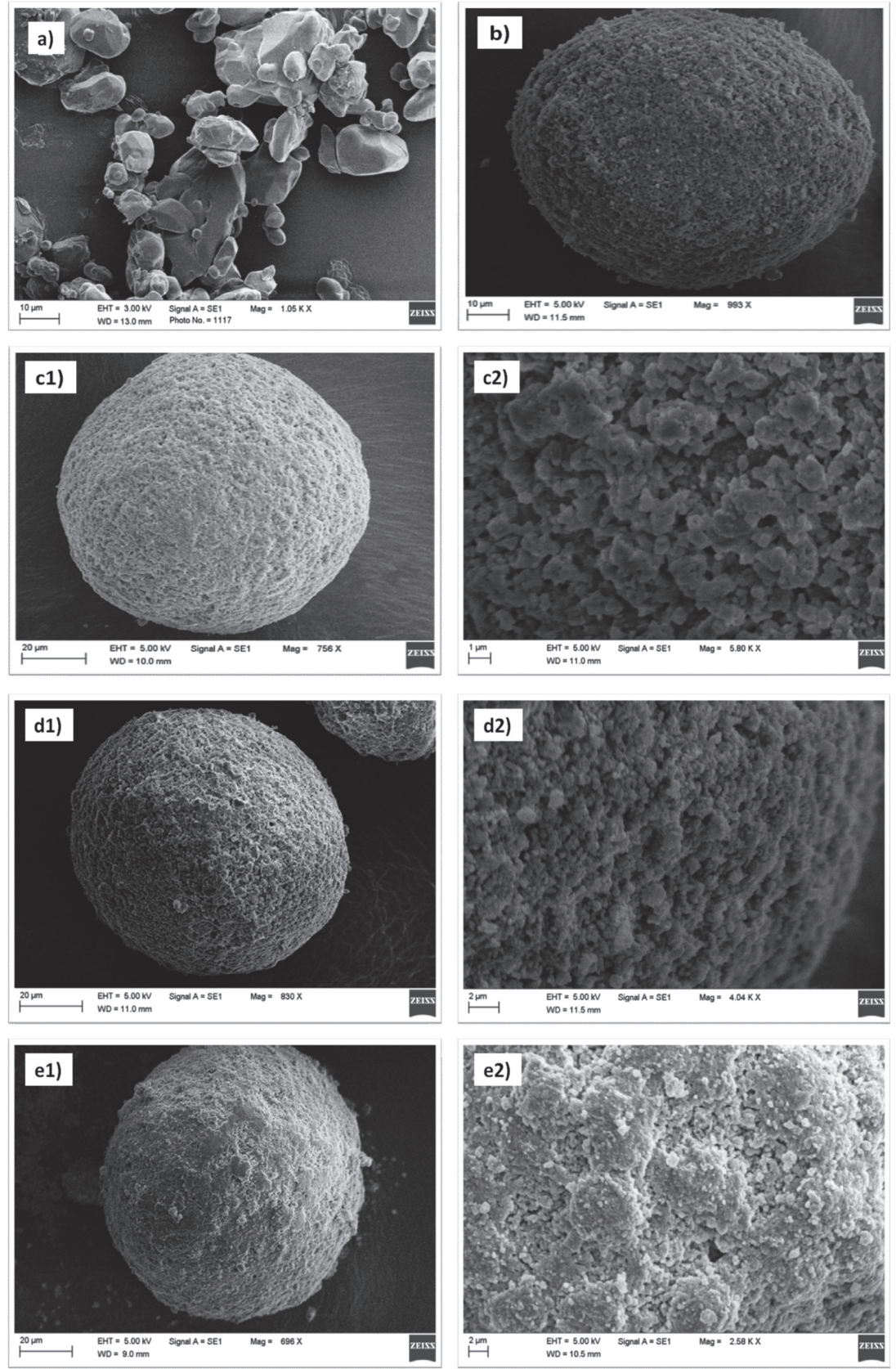

Fig. 3. Scanning electron micrographs of: a) pure fenofibrate, b) Neusilin US2 (NUS2) raw material, c) solid SEDDS-1/NUS2 50:50 \% $(\mathrm{m} / \mathrm{m})$, d) solid SEDDS-2/NUS2 50:50 \% $(\mathrm{m} / \mathrm{m})$, e) solid SNEDDS-3/NUS2 $50: 50 \%(\mathrm{~m} / \mathrm{m})$, at different magnifications. 
G. Shazly and K. Mohsin: Dissolution improvement of solid self-emulsifying drug delivery systems of fenofibrate using an inorganic high surface adsorption material, Acta Pharm. 65 (2015) 29-42.

Table V. Conversion of liquid formulations into solid SEDDS/SNEDDS using Neusilin US2 for in vitro dissolution studies

\begin{tabular}{ccc}
\hline LFCS type & Formulation No. & Drug dissolved in unit dose $(\% \mathrm{~m} / \mathrm{m})$ \\
\hline \multirow{2}{*}{ IIIA (solid SEDDS-1) } & F1 & 5.4 \\
& F2 & 6.4 \\
\multirow{2}{*}{ IIIA (solid SEDDS-2) } & F7 & 7.5 \\
& F3 & 6.3 \\
& F4 & 7.5 \\
IIIA (solid SNEDDS3) & F8 & 8.8 \\
& F5 & 4.2 \\
& F6 & 5.0 \\
\hline
\end{tabular}

SNEDDS formulations

of fenofibrate released from both fenofibrate raw powder and Lipanthyl ${ }^{\circledR}$ is very low (around 17 \%). In comparison, solid SEDDS-1 systems (F1, F2 and F7, Fig. 4) enhanced the dissolution rate of fenofibrate by $50 \%$ within 120 minutes.

Similarly, Fig. 4b compares the in vitro release profiles of fenofibrate from F3, F4, F8, control (fenofibrate raw powder) and Lipanthyl ${ }^{\circledR}$. It was found that formulations F3, F4 and F8 exhibited higher release (50-56 \%) compared to both the control and Lipanthyl (15\%). Regarding the release of fenofibrate from formulations F1, F2 and F7, it was found that formulation F1 (50 \% NUS2) showed higher release than formulations F2 and F7. This is evident from the similar results obtained when comparing fenofibrate release from F5, F6 and F9 (Fig. 4c).

The overall comparison of the in vitro dissolution profiles of fenofibrate from all solid SEDDS/SNEDDS (except F1, F2 and F7) was over $50 \%$ in $60 \mathrm{~min}$ and was significantly higher than the release from the control and marketed product Lipanthyl ${ }^{\circledR}(15 \%)$. In case of formulations F1, F2 and F7, the drug release was lower or delayed, which could be due to the water insoluble surfactant used in designing liquid SEDDS1 [(M812:I988 (7:3)/TO106V $(1 / 1)]$. The overall results of all the solid SEDDS/SNEDDS showed that the maximum percentage of drug release was achieved within $15 \mathrm{~min}$.

On the other hand, in vitro dissolution studies performed in the basic medium ( $\mathrm{pH} 7.4$ shown in Figs. 5a-c for the same formulations F1-F9, fenofibrate raw powder and Lipanthyl ${ }^{\circledR}$ are presented in Fig. 3. In case of formulations F1, F2 and F7, the drug release was faster and the dissolution efficiency was higher compared to the control and Lipanthyl ${ }^{\circledR}$. Cumulative percentage release from solid SEDDS (F1) was found to be more than $45 \%$, within 60 min and was significantly higher than that of the control and Lipanthyl ${ }^{\circledR}(17 \%)$. From the overall release data in the slightly basic medium it followed that all the formulations (F1-F9) showed higher release rate of fenofibrate compared to fenofibrate raw powder and Lipanthyl ${ }^{\circledR}$. In all cases of the solid SEDDS/SNEDDS, almost $45 \%$ of the drug was released within 15 min during the studies. Within all solid formulations, the use of $50 \%$ NUS2 (F1, F3 and F5) gave better fenofibrate release profiles.

The overall drug release profile of solid SEDDS and SNEDDS in the current investigation showed that all the developed formulations (F1-F9) had higher drug release profiles 
G. Shazly and K. Mohsin: Dissolution improvement of solid self-emulsifying drug delivery systems of fenofibrate using an inorganic high surface adsorption material, Acta Pharm. 65 (2015) 29-42.
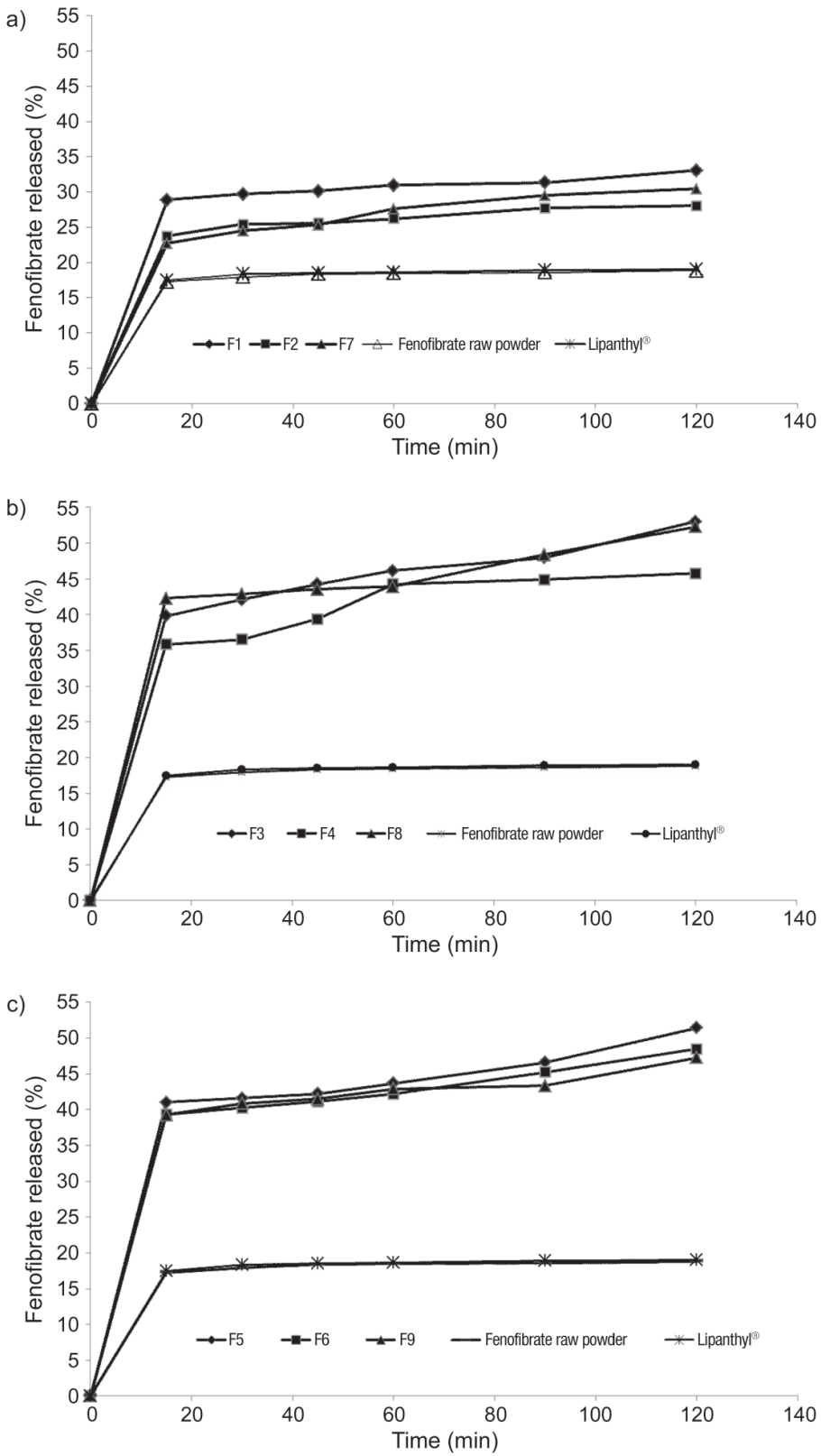

Fig. 4. In vitro release of fenofibrate from the representative LFCS type III formulation in $\mathrm{HCl} \mathrm{pH} 1.2$ at $37^{\circ} \mathrm{C}$ : a) solid SEDDS-1 (F1, F2, F7), control (fenofibrate raw powder) and marketed product (Lipanthy $\mathrm{l}^{\circledR}$ ), b) solid SEDDS-2 (F3, F4, F8), control (fenofibrate raw powder) and marketed product (Lipanthyl ${ }^{\circledR}$ ), c) solid SNEDDS3 (F5, F6, F9), control (fenofibrate raw powder) and marketed fenofibrate (Lipanthyl $\mathrm{l}^{\circledR}$ ). Mean values, $n=3$. 
G. Shazly and K. Mohsin: Dissolution improvement of solid self-emulsifying drug delivery systems of fenofibrate using an inorganic high surface adsorption material, Acta Pharm. 65 (2015) 29-42.
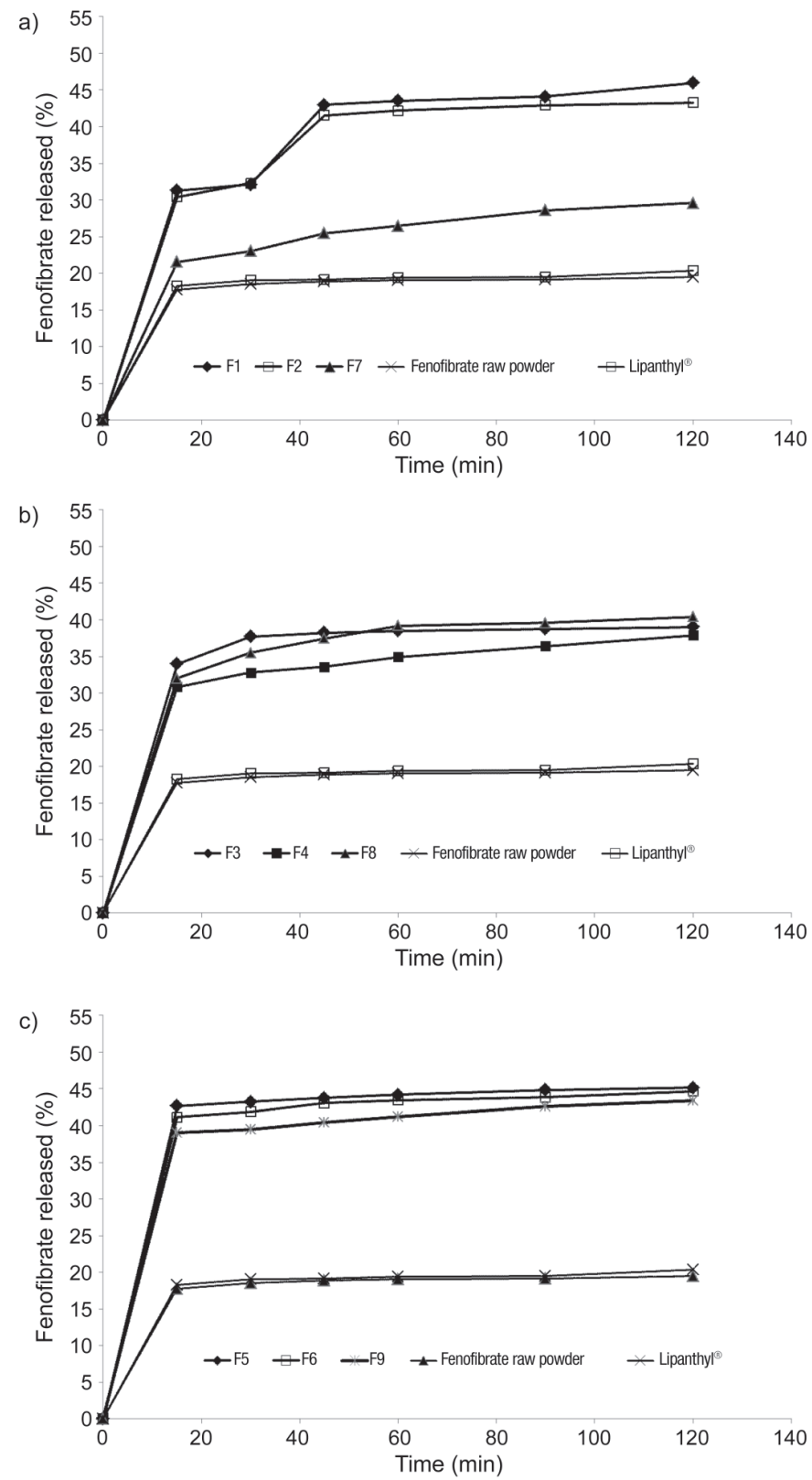

Fig. 5. In vitro release of fenofibrate from the representative LFCS type III formulation in phosphate buffer $\mathrm{pH} 7.4$ at $37^{\circ} \mathrm{C}$ : a) solid SEDDS-1 (F1, F2, F7), control (fenofibrate raw powder) and marketed product (Lipanthyl $\left.{ }^{\circledR}\right)$, b) solid SEDDS-2 (F3, F4, F8), control (fenofibrate raw powder) and marketed product (Lipanthyl ${ }^{\circledR}$ ), c) solid SNEDDS3 (F5, F6, F9), control (fenofibrate raw powder) and marketed fenofibrate $\left(\right.$ Lipanthyl $\left.{ }^{\circledR}\right)$. Mean values, $n=3$. 
than the fenofibrate raw powder and marketed product Lipanthyl ${ }^{\circledR}$. The results suggest that the solid SEDDS/SNEDDS formulation systems preserved the improvement of in vitro dissolution of liquid SEDDS/SNEDDS.

\section{CONCLUSIONS}

A large surface area material NUS2 with exceptional excipient properties for improving drug delivery was used as the microporous adsorbent to load SEDDE/SNEDDS. The solid state characterization studies indicate that the SEDDS2 and SNEDDS3 [(M812:1988 (7:3)/HCO30 (1/1), I308/HCO30 (1/1)] using 40-50 \% NUS2 proved to be free flowing fine powders in stable form and the potential systems for high drug loading.

All the solid SEDDS/SNEDDS showed enhanced dissolution profile, compared to both fenofibrate raw powder and Lipanthyl ${ }^{\circledR}$. Solid state characterization employing the SEM, XRD and DSC studies indicated that the SEDDS/SNEDDS powder produced smooth granular particles in amorphous form.

In conclusion, solidification using adsorbents is a powerful technique for the preparation of rapidly dissolving formulations of fenofibrate. The solidification of SEDDS/SNEDDS using NUS2 improved the surface adsorption, porosity, anticaking flow enhancing properties and the ability to keep the drug stable in amorphous state, which could be one of the best carriers today to deliver poorly soluble drugs.

This approach could potentially lead to better bioavailability of fenofibrate drug products. Conversion of a liquid self-emulsifying drug delivery system into a free flowing solid powder using high surface inorganic materials (Neusilin US2) is one of the most recent and promising approaches in pharmaceutical dosage systems. However, knowledge of the integrity of excipients is helpful in this regard, but it is ultimately necessary to test each formulation.

The influence of »drug-adsorbent formulation« interactions should be investigated on the case-by-case basis in any formulation.

Acknowledgements. - The author extends his appreciation to the Deanship of Scientific Research at King Saud University for funding the work through the research group project No. RGP-VPP-139.

\section{REFERENCES}

1. C. W. Pouton, Formulation of poorly water-soluble drugs for oral administration: physicochemical and physiological issues and the lipid formulation classification system, Eur J. Pharm. Sci. 29 (2006) 278-287; DOI: 10.1016/j.ejps.2006.04.016.

2. C. Porter, C. Pouton, J. Cuine and W. Charman, Enhancing intestinal drug solubilisation using lipid-based delivery systems, Adv. Drug Deliv. Rev. 60 (2008) 673-691; DOI: 10.1016/j.addr.2007.10.014.

3. K. Mohsin, M. A. Long and C. W. Pouton, Design of lipid-based formulations for oral administration of poorly water-soluble drugs: precipitation of drug after dispersion of formulations in aqueous solution, J. Pharm. Sci. 98 (2009) 3582-3595; DOI: 10.1002/jps.21659.

4. A. A. Shahba, K. Mohsin and F. K. Alanazi, Novel self-nanoemulsifying drug delivery systems (SNEDDS) for oral delivery of cinnarizine: design, optimization, and in-vitro assessment, AAPS PharmSciTech. 13 (2012) 967-77; DOI: 10.1208/s12249-012-9821-4. 
5. A. J. Humberstone and W. N. Charman, Lipid based vehicles for the oral delivery of poorly water soluble drugs, Adv. Drug Deliv. Rev. 25 (1997) 103-128; DOI: 10.1016/s0169-409x(96)00494-2.

6. C. M. O'Driscoll and B. T. Griffin, Biopharmaceutical challenges associated with drugs with low aqueous solubility-The potential impact of lipid-based formulations, Adv. Drug Deliv. Rev. 60 (2008) 617-624; DOI: 10.1016/j.addr.2007.10.012.

7. B. Singh, S. Beg, R. K. Khurana, P. S. Sandhu, R. Kaur and O. P. Katare, Recent advances in selfemulsifying drug delivery systems (SEDDS), Crit. Rev. Ther. Drug Carrier Syst. 31 (2014) 121-185; DOI: 10.1615/ CritRevTherDrugCarrierSyst. 2014008502.

8. L. Wang, J. Dong, J. Chen, J. Eastoe and X. Li, Design and optimization of a new self-nanoemulsifying drug delivery system, J. Colloid Interf. Sci. 330 (2009) 443-448; DOI: 10.1016/j.jcis.2008.10.077.

9. G. Rossi, P. Reggiani, M. B. Regazzi, S. Gatti, F. Ceccherelli, P. Prato, D. Galmarini and L. R. Fassati. Neoral increases bioavailability versus oral sandimmune after porcine small bowel transplantation, Transplant. Proc. 30 (1998) 2654-2656; DOI: 10.1016/S0041-1345(98)00776-3.

10. K. Mohsin, Design of lipid-based formulations for oral administration of poorly water-soluble drug fenofibrate: effects of digestion, AAPS PharmSciTech. 13 (2012) 637-646; DOI: 10.1208/s12249012-9787-2.

11. R. P. Ashok and R. V. Pradeep, Preparation and in vivo evaluation of SMEDDS (self-microemulsifying drug delivery system) containing fenofibrate, AAPS J. 9 (2007) 344-352; DOI: 10.1208/aapsj0903041.

12. A. Abdallah, S. Klein and K. Mader, A new self-emulsifying drug delivery system (SEDDS) for poorly soluble drugs: characterization, dissolution, in vitro digestion and incorporation into solid pellets, Eur. J. Pharm. Sci. 35 (2008) 457-464; DOI: 10.1016/j.ejps.2008.09.006.

13. S. Nazzal and M. Khan, Controlled release of a self-emulsifying formulation from a tablet dosage form: Stability assessment and optimization of some processing parameters, Int. J. Pharm. 315 (2006) 110-121; DOI: 10.1016/j.ijpharm.2006.02.019.

14. C. M. Perry and S. Noble, Saquinavir soft-gel capsule formulation. A review of its use in patients with HIV infection, Drugs 55 (1998) 461-486; DOI: 10.2165/00003495-199855030-00014.

15. B. Tang, G. Cheng, J. C. Gu and C. H. Xu, Development of solid self-emulsifying drug delivery systems: preparation techniques and dosage forms, Drug Discov. Today 13 (2008) 606-612; DOI: 10.1016/j.drudis.2008.04.006.

16. C. W. Pouton, Lipid formulations for oral administration of drugs: non-emulsifying, self-emulsifying and 'self-microemulsifying' drug delivery systems, Eur. J. Pharm. Sci. 11 (Suppl 2) (2000) S93-S98; DOI: 10.1016/S0928-0987(00)00167-6.

17. V. Agarwal, A. Siddiqui, H. Ali and S. Nazzal, Dissolution and powder flow characterization of solid self-emulsified drug delivery system (SEDDS), Int. J. Pharm. 366 (2009) 44-52; DOI: 10.1016/j. ijpharm.2008.08.046.

18. R. N. Gursoy and S. Benita, Self-emulsifying drug delivery systems (SEDDS) for improved oral delivery of lipophilic drugs, Biomed. Pharmacother. 58 (2004) 173-182; DOI: 10.1016/j.biopha.2004.02.001.

19. E. Atef and A. Belmonte, Formulation and in vitro and in vivo characterization of a phenytoin self-emulsifying drug delivery system (SEDDS), Eur. J. Pharm. Sci. 35 (2008) 257-263; DOI: 10.1016/j. ejps.2008.07.004.

20. T. R. Kommuru, B. Gurley, M. A. Khan and I. K. Reddy, Self-emulsifying drug delivery systems (SEDDS) of coenzyme Q10: formulation development and bioavailability assessment, Int. J. Pharm. 212 (2001) 233-246; DOI: 10.1016/S0378-5173(00)00614-1.

21. R. L. Carr, Evaluating flow properties of solids, Chem. Eng. 72 (1965) 163-168. 